\title{
Into the fission valley of 'magic nucleus' polonium
}

\author{
Tilak Kumar Ghosh
}

The word 'radioactive' was first coined by Marie Curie when she, along with her husband Pierre Curie, discovered the element Polonium (Po). The nucleus ${ }^{210}$ Po is a testing ground for many theoretical and experimental aspects of nuclear structure as well as nuclear fission dynamics as it is a 'magic nucleus' with neutron number $N=126$. At Variable Energy Cyclotron Centre, Kolkata, the fission of Polonium nuclei is being studied in order to understand the survival of nuclear shell effects that is known to be the key for the stability of super heavy elements (SHE).

Keywords: Mass distribution, nuclear fission, shell effects, super heavy elements.

THE year 2018 was celebrated as the 150th birth anniversary of Marie Skłodowska-Curie (1867-1934). She was the first woman to win a Nobel Prize and the first scientist to win two; one in the field of Physics (1903) and the other in Chemistry (1911). Polonium nuclei were discovered by Marie Curie and her husband Pierre Curie ${ }^{1,2}$. The substance that Curie couple extracted from pitchblende contained an unknown element, similar to bismuth in its analytical properties but much more active than uranium, the only active element known at that time. They proposed that the new element be named Polonium (Po) in honour of Poland, the native of Marie Curie.

Polonium is also an interesting element to nuclear physicists. One of its isotopes, ${ }^{210}$ Po has neutron number $N=126$ which is a magic number. The nuclei with the magic number of nucleons (neutron or proton) show extra stability as the outer nuclear shell is fully filled up. This is known as nuclear shell effect. A close analogy of magic nuclei is inert gases (helium, neon, argon, etc.) which are chemically inert because of their outermost electronic orbits being completely filled up. Usually with increase in atomic (proton) number, nuclei become unstable because of coulomb repulsion. However, it is predicted that due to the nuclear shell effect, elements having atomic number higher than 104, called super heavy elements (SHE), do exist. The current chart of the periodic table is being extended with newly discovered $\mathrm{SHE}^{3}$. Theoreticians predict that the SHE with next neutron magic number $N=184$ and proton number $Z=114 / 120 /$ 126 (they have different opinion about the proton numbers) will be a stable element. Efforts are on worldwide to reach this island of stability ${ }^{4,5}$.

Tilak Kumar Ghosh is in the Variable Energy Cyclotron Centre, 1/AF, Bidhannagar, Kolkata 700 064, India and Homi Bhabha National Institute, Training School Complex, Anushakti Nagar, Mumbai 400 094, India. e-mail: tilak@vecc.gov.in
However, to produce SHE one would require to find out the best combinations of nuclei (target and projectile) to be fused in the laboratory ${ }^{6,7}$. Also it is required to be ensured that the SHEs are produced at right excitation energies at which nuclear shell effects survives ${ }^{8}$. Since currently it is not possible to produce the element with $N=184$ (as that would require highly neutron-rich targets and projectiles which are not available in nature), ${ }^{210} \mathrm{Po}$ with magic number $N=126$ has been studied extensively to explore the survival of nuclear shell effects at the excitation energy of around $40 \mathrm{MeV}$, beyond which one would expect the shell effects to vanish. Researchers at the Variable Energy Cyclotron Centre (VECC), Kolkata carried out several experiments recently ${ }^{9,10}$ to explore the existence of shell effects at fission saddle at moderate excitation energy for ${ }^{210} \mathrm{Po}$.

A few recent experimental and theoretical studies ${ }^{11-14}$, however, indicated considerable ambiguity regarding the presence or absence of shell effects (correction) at the saddle point. Saddle point is the point of no return, so that a nucleus, if deformed beyond this point, will have no choice but to undergo fission, leading to two heavy fragments of nearly equal mass. Deviation of fission fragment's angular anisotropy (which is the ratio of fission yields along the beam direction and perpendicular to the beam direction) from theory was observed in the fission of ${ }^{210} \mathrm{Po}$, produced in nuclear reaction ${ }^{12} \mathrm{C}+{ }^{198} \mathrm{Pt}$, at excitation energy of $\sim 40-60 \mathrm{MeV}$. This was conjectured as an indirect evidence of shell effect at saddle due to neutron shell closure at $N=126$ (ref. 13).

During the fission process, many neutrons are also emitted, both before and after the scission. The neutrons emitted prior to fission are called the pre-scission neutrons. They are likely to carry signature of the dynamics of the process and therefore can be used as an independent experimental probe, similar to angular anisotropy. The pre-scission neutron multiplicity was measured in an 


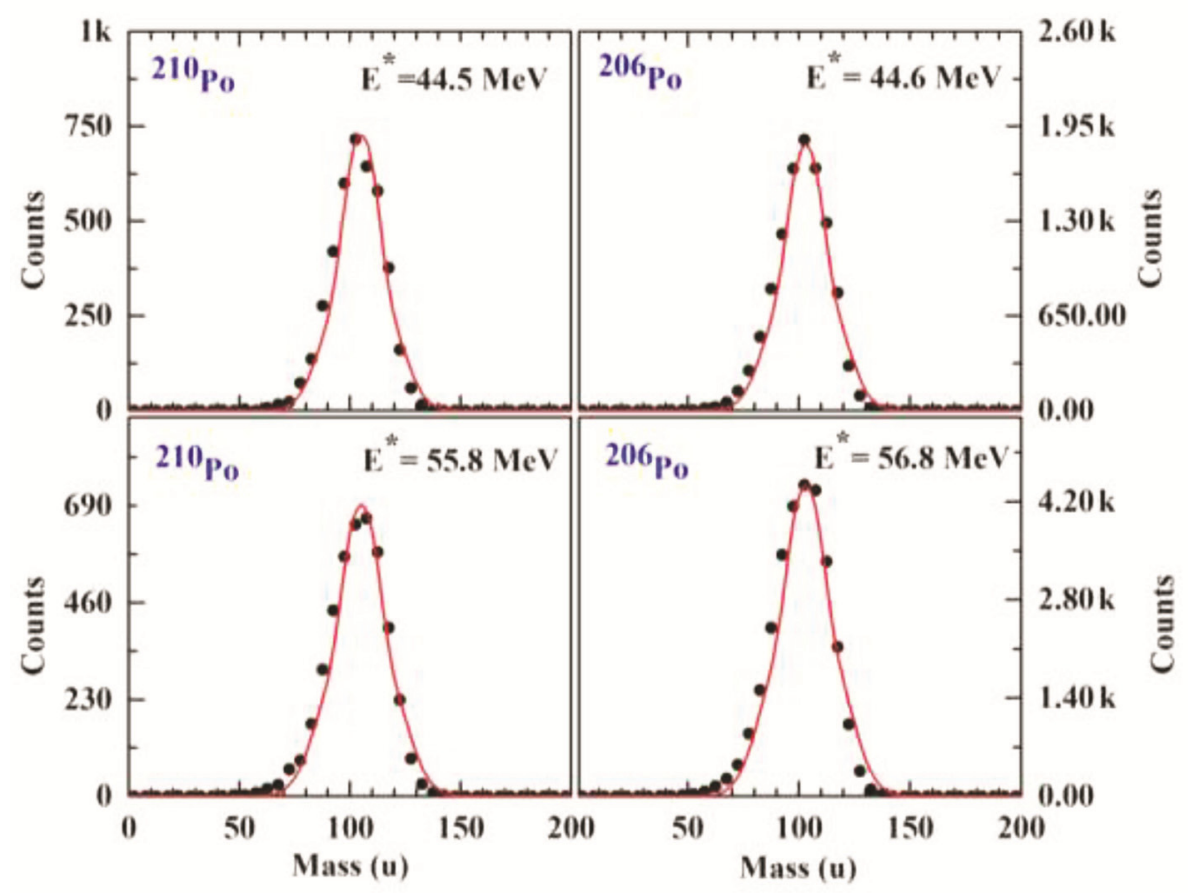

Figure 1. Measured mass distributions of fission fragments at similar excitation energies for the two polonium isotopes. At these excitation energies, the mass distributions are single peaked and could be fitted by a single Gaussian distribution as shown by solid (red online) lines.

experiment at the Inter University Accelerator Centre (IUAC), New Delhi, India, for ${ }^{206,210}$ Po. The result also indicated $^{12}$ the requirement of substantial shell correction not only in shell closed ${ }^{210} \mathrm{Po}$ but also in ${ }^{206} \mathrm{Po}$. The analysis of both the angular anisotropy and pre-scission neutron data mentioned above, were carried out within the framework of well-established statistical models. However, Schmitt et al. ${ }^{11}$ with dynamical calculations using stochastic Langevin equation, claimed that the angular anisotropy and neutron data (mentioned above), could well be explained with a purely macroscopic potential energy landscape without considering any shell effect at saddle point. Recent statistical model calculation ${ }^{15}$ carried out by the scientists of Bhabha Atomic Research Centre, Mumbai, India for the nucleus ${ }^{210}$ Po populated in light and heavy ion induced reaction, could describe the excitation functions without the requirement of shell correction at saddle, but required a huge fission delay to fit the prescission neutron multiplicity data in heavy ion induced reaction.This prevailing noticeable ambiguity necessitated an immediate evaluation of the problem through a new experimental observation.

The fission fragment mass distributions can be used as an experimental probe to look for the presence or absence of shell effects in nuclei ${ }^{8}$. Therefore mass distributions of the nuclei ${ }^{210,206} \mathrm{Po}$ were studied ${ }^{9}$ by the researchers at VECC. The nuclei were populated by bombarding ${ }^{12} \mathrm{C}$ projectile from Tata Institute of Fundamental ResearchBhabha Atomic Research Centre pelletron machine on ${ }^{198,194} \mathrm{Pt}$ targets. Large area multi-wire proportional coun- ters (MWPC) ${ }^{16}$ developed at VECC were used in the experiment to detect the fission fragments. Variations of the width of the fission fragment mass distributions with excitation energies were studied in the experiment.

The presence of shell effect would make the fission mass distributions either double humped or wider (compared to the theoretical estimate). Figure 1 shows the typical measured mass distributions at similar excitation energies for both the nuclei ${ }^{210} \mathrm{Po}$ and ${ }^{206} \mathrm{Po}$. The mass distributions could be well fitted by a single Gaussian as shown by the red line. Variation of the standard deviation $(\sigma)$ of the fitted mass distributions with excitation energy, shown in Figure 2, was found to increase monotonically, consistent with theoretical estimate without incorporation of shell effect at the saddle point. This clearly indicated the absence of shell correction at the saddle point in both the polonium isotopes up to $40 \mathrm{MeV}$ excitation energy. The result of a previous experiment ${ }^{9}$ provides benchmark data to test new fission dynamical models to study the effect of shell correction on the potential energy surface at fission saddle point.

After the discovery ${ }^{17}$ of nuclear fission, one of the biggest challenges was to explain the observation of asymmetric (double hump) mass distribution by Lise Meitner ${ }^{18}$, while the liquid drop model ${ }^{19}$ predicted symmetric mass distribution. The asymmetry in mass distribution was understood by considering fragment shell properties $^{20}$. It was conjectured that the 2 nd peak (around mass number 132) of the mass distribution was due to the doubly magic shell configuration. Thus, it was believed 
for years that shell effects of the fission fragments were responsible for this asymmetric mass distribution. However, a recent experiment carried out at CERN ISOLDE facility changed this notion.

In the pioneering experiment carried out at $\mathrm{CERN}^{21}$, fragment mass distribution of beta delayed fission of ${ }^{180} \mathrm{Hg}$ was measured. The excitation energy of the nucleus was low $(\sim 10 \mathrm{MeV})$. At this excitation energy, shell effect is expected to survive. One would also expect that the mass distribution of ${ }^{180} \mathrm{Hg}$ be symmetric (peaking around mass number 90 , with semi magic proton number $Z=40$ and neutron number $N=50$ ), if the shell effects of the fission fragments are to decide the fate of the mass distribution. However, the measured mass distribution was found to be asymmetric, peaking around mass number 80 and 100 (neither of which is near magic number), clearly contradicting the expectation.

Several theories came up to explain the puzzle of asymmetric mass distribution of ${ }^{180} \mathrm{Hg}$ (refs 22, 23).

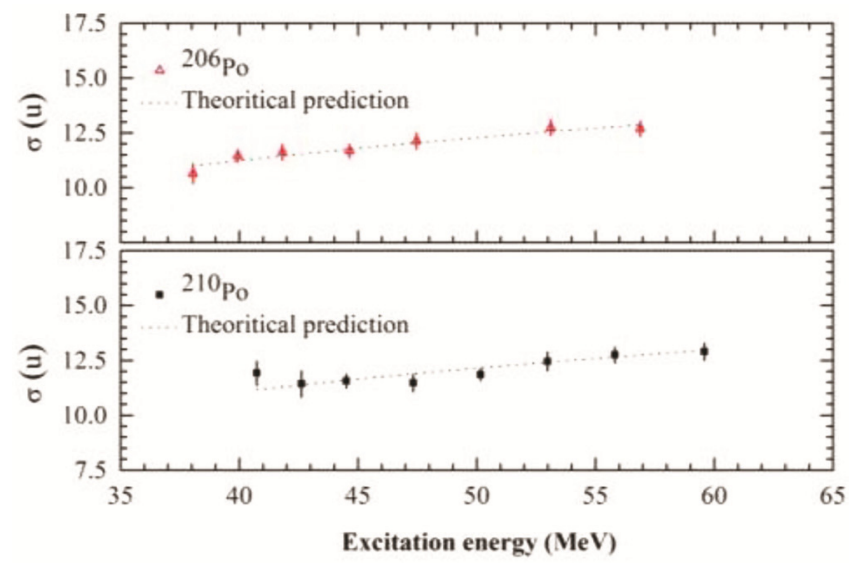

Figure 2. Variation of the standard deviation $(\sigma)$ of the fitted symmetric mass distributions with excitation energy. The calculated standard deviations from the previous study ${ }^{9}$ are shown by dotted lines.

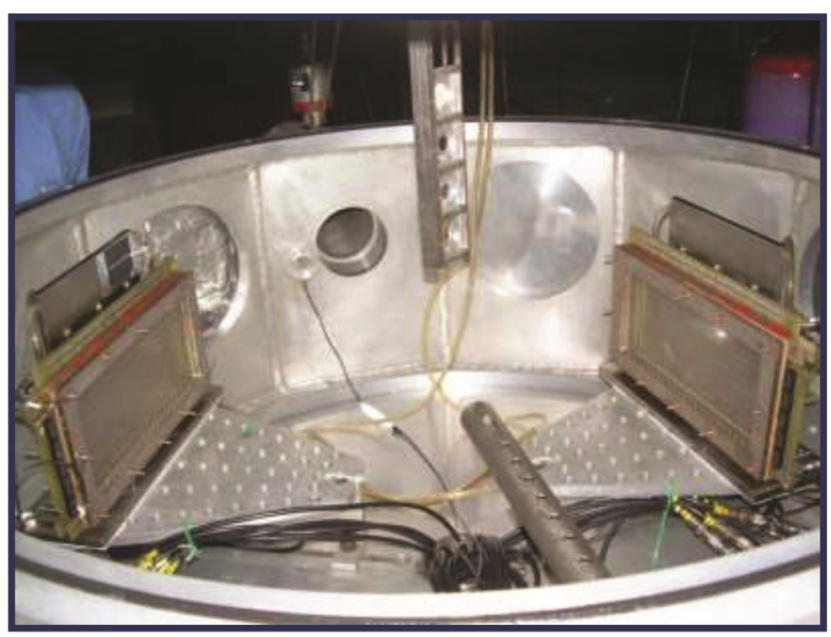

Figure 3. Photograph of the MWPC detectors mounted inside a scattering chamber for the detection of fission fragments.
These theories claim that apart from the shell effects of the fission fragments, the shell effects during transition from saddle to scission (known as fission valley) play a decisive role in deciding the fission fragment mass distribution. The macroscopic-microscopic model (Brownian shape motion on five-dimensional (5D) potential energy surface) of Moller and Randrup ${ }^{22}$ could successfully explain the asymmetric mass distribution of ${ }^{180} \mathrm{Hg}$. But, a good theory should be capable of predicting mass distributions of several nuclei. In this regard Moller's theory predicted asymmetric mass distribution for ${ }^{210} \mathrm{Po}$ nuclei at an excitation energy of $31.43 \mathrm{MeV}$, while at higher excitation energies mass distributions were predicted to be symmetric.

In order to test these theories, in a recent experiment conducted at the VECC K-130 cyclotron, alpha beam was bombarded on an enriched ${ }^{206} \mathrm{~Pb}$ target to populate the ${ }^{210} \mathrm{Po}$ nuclei. At lower excitation energies, fission cross sections were low (few milli barn). However, the large area MWPC indigenously developed at VECC, that provides large detection coverage, helped us to successfully measure such a low cross section. A photograph of the experimental setup is shown in Figure 3. The masses of the fission fragments were calculated from the time of flight difference of the fragments and their positions (theta, phi) measured with the MWPCs.

The measured fragment mass distributions ${ }^{10}$ for the reaction ${ }^{4} \mathrm{He}+{ }^{206} \mathrm{~Pb}$ populating ${ }^{210} \mathrm{Po}$ were found to be symmetric and the width of the mass distributions were

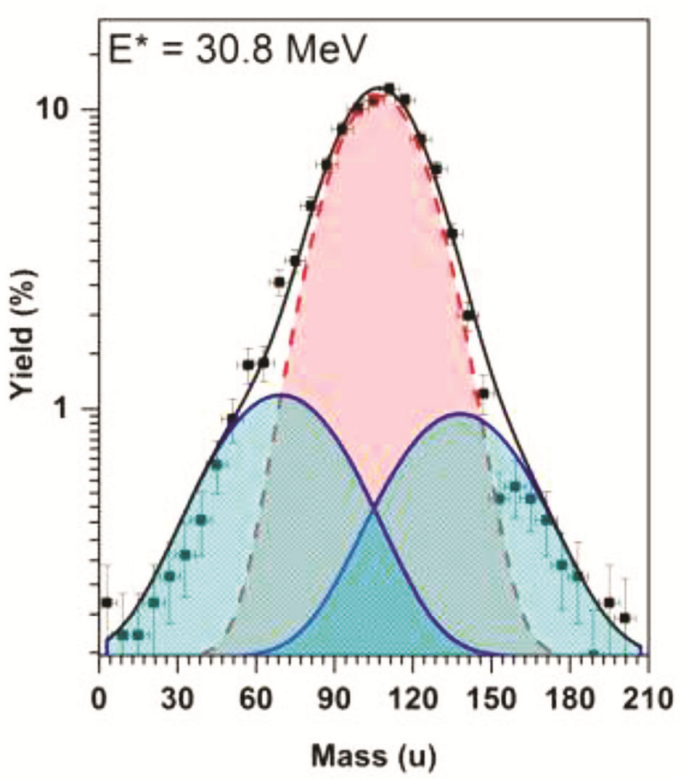

Figure 4. Asymmetric mass distributions observed in the fission of ${ }^{210}$ Po nuclei at the excitation energy $30.8 \mathrm{MeV}$. This verifies a recent theoretical calculation ${ }^{20}$ to predict the mass distribution of polonium and other nuclei (e.g. ${ }^{180} \mathrm{Hg}$ ). The red dashed line indicates the Gaussian fit corresponding to the symmetric fragments, while the blue solid lines show the asymmetric components. The overall fitting is shown by the solid black line. 
found to increase monotonically with excitation energy above $36 \mathrm{MeV}$, indicating the absence of shell effect at the saddle. However, deviation from symmetric mass distributions at the lowest excitation energy $(30.8 \mathrm{MeV})$ was indeed observed as shown in Figure 4. At this excitation energy, a weak asymmetric component $(\approx 1 \%$ yield level) was shown to coexist along with the strong symmetric mass distribution. The heavier mass side of the asymmetric distribution was observed to peak around $\approx 132$ (doubly magic ${ }^{132} \mathrm{Sn}$ ), a clear indication of the persistence of the shell effect of the nascent fragments. VECC experimental data ${ }^{10}$ reaffirmed the theory of Moller and Randrup $^{22}$.

From the theoretical standpoint, fission process is, in general, successfully explained using statistical model without any explicit inclusion of the dynamical features in the theory. The effect of dynamics in fission has been implicitly taken care of in this model by the introduction of an adjustable parameter called 'fission delay time'. However, inadequacy of these models, even after the inclusion of the phenomenology of fission delay, was clearly revealed in a few recent, novel, high precision experiments $^{10,21}$, which called for new generation of microscopic dynamical theories to properly explain these experiments. With the capability of precision calculation of 5D potential energy surface over the whole fission landscape, dynamical theories are now all set to dominate the fission show and provide next insights into the next generation of fission experiments in the coming years. However, statistical models, being for just simple computational use compared to dynamical models, are also likely to remain equally important to provide general interpretation of the fission process.

It is clear from the above discussions that the shell effect on the evolution of fission dynamics is still not fully understood. The polonium case presented here showed a classic example of different facets of shell effects unfolded through the use of different experimental probes. The experimental fission research programme at VECC to measure the fission fragment mass distributions of polonium nuclei, firmly supports the fact that the shell effects at fission valleys (i.e. during transition from saddle to scission) play an important role. This indeed changes our notion that it is only the shell effects of the fission fragments that decide the fate of the mass distributions.

Polonium was discovered by the Curie couple. For Marie Curie's 150th birth anniversary, we nuclear physicists offer our tribute, for her important discovery of the element which still plays an important role in the frontier area of nuclear physics research.

1. Sklodowska-Curie, M., C. R. Acad. Sci., 1898, 126, 1101

2. Curie, P. and Curie, M., C. R. Acad. Sci., 1898, 127, 175.

3. Van Noorden, R., Four chemical elements added to periodic table. Nature, 2016; doi:10.1038/nature.2016.19112.
4. Oganessian, Y. T. and Utyonkov, V. K., Super-heavy element research. Rep. Prog. Phys., 2015, 78, 036301.

5. Düllmann, C. E. and Block, M., Island of heavy weights. Sci. Am., March 2018, 42.

6. Banerjee, K., et al., Fission dynamics study in ${ }^{243} \mathrm{Am}$ and ${ }^{254} \mathrm{Fm}$. Phys. Rev. C, 2016, 93, 064602.

7. Chaudhuri, A. et al., Fission fragment mass distributions in reactions populating ${ }^{200} \mathrm{~Pb}$. Phys. Rev. C, 2016, 94, 024617.

8. Chaudhuri, A. et al., Direct evidence of washing out of nuclear shell effects. Phys. Rev. C, 2015, 91, 044620.

9. Chaudhuri, A. et al., No influence of a $N=126$ neutron-shell closure in fission-fragment mass distributions. Phys. Rev. C, 2015, 92, 041601(R).

10. Sen, A. et al., Fission fragment mass distributions from ${ }^{210} \mathrm{Po}$ and ${ }^{213}$ At. Phys. Rev. C, 2017, 96, 064609.

11. Schmitt, C., Mazurek, K. and Nadtochy, P. N., On current ambiguity in the interpretation of fission at intermediate excitation energy. Phys. Lett. B, 2014, 737, 289.

12. Golda, K. S. et al., Determination of shell correction energies at saddle point using pre-scission neutron multiplicities. Nucl. Phys. A, 2013, 913, 157.

13. Shrivastava, A. et al., Shell effects in fission fragment anisotropies for ${ }^{12} \mathrm{C}+{ }^{194,198} \mathrm{Pt}$ systems. Phys. Rev. Lett., 1999, 82, 699.

14. Mahata, K. and Kailas, S., Re-examination of fission in the $A \approx 200$ mass region with excitation energy near $50 \mathrm{MeV}$. Phys. Rev. C, 2017, 95, 054616 .

15. Mahata, K., Kailas, S. and Kapoor, S. S., Fission barrier, damping of shell correction, and neutron emission in the fission of $A \sim 200$. Phys. Rev. C, 2015, 92, 034602 and references therein.

16. Ghosh, T. K. et al., Time of flight (TOF) spectrometer for accurate measurement of mass and angular distribution of fission fragments in heavy ion induced fission reactions. Nucl. Instrum. Methods Phys. Res. A, 2005, 540, 285.

17. Hahn, O. and Strassmann, F., Naturwissenschaften, 1939, 27(1), 11.

18. Meitner, L. and Frisch, O. R., Disintegration of uranium by neutrons: A new type of nuclear reaction. Nature (London), 1939, 143, 239.

19. Bohr, N. and Wheeler, J. A., The mechanism of nuclear fission. Phys. Rev., 1939, 56, 426.

20. Moller, P. and Nilsson, S. G., The fission barrier and odd multipole shape distortions. Phys. Lett., 1970, 31B, 283.

21. Andreyev, A. N. et al., New type of asymmetric fission in protonrich nuclei. Phys. Rev. Lett., 2010, 105, 252-502.

22. Moller, P. and Randrup, J., Calculated fission-fragment yield systematics in the region $74 \leq Z \leq 94$ and $90 \leq N \leq 150$. Phys. Rev. $C$, 2015, 91, 044316.

23. McDonnell, J. D., Nazarewicz, W., Sheikh, J. A., Staszczak, A. and Warda, M., Excitation-energy dependence of fission in the mercury region. Phys. Rev. C, 2014, 90, 021302(R).

ACKNOWLEDGEMENTS. The article is dedicated to Prof. Sailajananda Bhattacharya (former Head of Physics Group, VECC) on the eve of the completion of his tenure as a Raja Ramanna Research Fellow as a gesture of gratitude to his valuable encouragement and support. I acknowledge the contributions of all his collaborators and accelerator staffs at VECC, Kolkata and BARC-TIFR Pelletron facility, Mumbai, and IUAC, New Delhi. I also thank his students, Abhirup Chaudhuri, Trinath Banik, Arnab Ghosh and colleagues, C. Bhattacharya, Arijit Sen, K. Banerjee, S. Kundu, T. K. Rana, J. K. Meena, G. Mukherjee, P. Roy, R. Pandey, S. Manna, J. K. Sahoo, A. Saha, R. Saha Mondal.

Received 21 March 2018; revised accepted 1 April 2019

doi: $10.18520 / \mathrm{cs} / \mathrm{v} 116 / \mathrm{i} 12 / 1961-1964$ 